\title{
RM28
}

\section{Uncertainty Identification for Improved Reservoir Characterization and Field Development Planning}

\section{R.L. Vaughan* (ExxonMobil Upstream Research Company) \& A.M. Apanel (ExxonMobil Production Company)}

\section{SUMMARY}

Successful reservoir modeling projects share at least one important feature: a clear, concise, and businessrelevant purpose. This is especially true in today's competitive environment, where speed and efficiency are increasingly valued to make quicker decisions and capture opportunities. Models are tools designed to answer questions and, depending on the validity of the geological interpretation, quality of the input data, and availability of calibration data, may not be able to test the full range of uncertainties. Multi-functional uncertainty identification (UI) is the key to focusing these questions. In this talk, real-life workflows and examples are used to describe efficient methods for identifying, incorporating, and using uncertainty analysis. 
Successful reservoir modeling projects share at least one important feature: a clear, concise, and business-relevant purpose. This is especially true in today's competitive environment, where speed and efficiency are increasingly valued to make quicker decisions and capture opportunities. Models are tools designed to answer questions and, depending on the validity of the geological interpretation, quality of the input data, and availability of calibration data, may not be able to test the full range of uncertainties. Multi-functional uncertainty identification (UI) is the key to focusing these questions.

Successful reservoir UI, characterization and modeling benefit from certain behaviours:

- Very early UI that is revisited throughout the characterization-modeling process and driven by:

- Commercial-Engineering-Geoscience workshops to identify uncertainties

- Early incorporation of production surveillance and/or analogue data

- Early identification of competing concepts and scenarios to avoid "anchoring" on a the most-likely interpretation

- Tailoring the process to the asset and business phase such as:

- Type and complexity of the reservoir and fluids

- Capital-intensive new field development vs. incremental projects in mature producing assets

- Recognition of "Irreducible Uncertainty" resulting in:

- Elimination of superfluous work and better risk mitigation planning

- Use of end-member sensitivity analysis, including identification of breakover/threshold points to better manage potential impacts to the project

- Determination of, and alignment on, what not to work

- Identification of project "show-stoppers" with an efficient plan to address each leading to:

- Appropriate choice of interpretation products and model types

$\circ$ Early exploration of boundaries and sensitivities

Some of these behaviours may require a change in corporate culture. For UI and modeling to have a significant impact on, for example, the development concept and facilities-sizing for a new field, results need to be provided much earlier than traditional complex and deterministic full-field models allow. Simpler models with potentially reduced conditioning to well and seismic data should be able to address the key development decisions up front and guide later development of more detailed models to optimize the depletion plan. A consistent and structured approach for collecting and presenting uncertainty data at all project stages is also required for communication with internal and external decision makers.

UI should ideally begin with characterization, well in advance of the geological modeling process. Especially important will be the identification of key concerns (show-stoppers) and features that may materially impact volumes, fluid flow and project economics. In addition, gaps or other uncertainties in the input data need to be identified and understood. Simple box or sector models at this stage may be useful to judge the impact of various parameters and thus guide the degree of characterization required. Sensitivity analysis can identify "break-over" points- the threshold at which a parameter begins to materially affect an outcome - and these may help refine the characterization process.

Ultimately, all the uncertainty analysis results should lead to decisions to proceed, walk-away, obtain more data and/or refine risk mitigation planning.

The best examples of efficient UI and modeling processes typically involve compressed-schedule evaluations such as for farm-outs, asset sales, or bidding rounds. Compressed time-frames override standard, detailed modelling workflows and force all parties to quickly "get to the heart of the matter." In these situations, multi-functional teams of experienced and advanced experts typically distil the issues down to a few key items that can be managed through the use of judgment-based methods involving analogues, simple decline curve or material balance analyses, and strip, sector or 
well models. A key challenge to reservoir modeling today is how to take the best aspects of these accelerated evaluations and apply them successfully to the larger and more common major projects. Success will invariably include the adoption of early, structured uncertainty identification and the acceptance of multiple small models and simpler full-field models. 